\title{
Regional Geomagnetic Field Model for Croatia at 2009.5
}

\author{
Eugen VUJIĆ ${ }^{1}$, Mario BRKIĆ ${ }^{1}$, and Peter KOVÁCS ${ }^{2}$ \\ ${ }^{1}$ Faculty of Geodesy, University of Zagreb, Zagreb, Croatia \\ e-mails: eugvujic@gfz.hr (corresponding author), mbrkic@geof.hr \\ ${ }^{2}$ Geological and Geophysical Institute of Hungary, Budapest, Hungary \\ e-mail: kovacs.peter@mfgi.hu
}

\begin{abstract}
Geomagnetic data of north, east, and vertical components at Croatian repeat stations and ground survey sites, as well as European geomagnetic observatories and repeat stations, were used to obtain a regional geomagnetic model over Croatia at 2009.5 epoch. Different models were derived, depending on input data, and three modelling techniques were used: Taylor Polynomial, Adjusted Spherical Harmonic Analysis, and Spherical Harmonic Analysis. It was derived that the most accurate model over Croatia was the one when only Croatian data were used, and by using the Adjusted Spherical Harmonic Analysis. Based on Croatian repeat stations' data in the interval 2007.5-2010.5, and a global Enhanced Magnetic Model, it was possible to estimate the crustal field at those sites. It was also done by taking into account the empirical adjustment for long-term external field variations. The higher crustal field values were found at those stations which are on or close to the Adriatic anomaly.
\end{abstract}

Key words: geomagnetic repeat stations, geomagnetic field model, crustal field. 


\section{INTRODUCTION}

The geomagnetic field at the Earth's surface is a superposition of several internal and external field contributions, which occur on different spatial and temporal scales. After the removal of external field contributions and their induced effects, there remains only the internal field, which consists of the core and lithospheric field. The lithospheric field varies with a shorter spatial scale compared to the more regular spatial trend of the core field, and is consequently mainly masked by it (Thébault et al. 2006, 2010; Korte and Thébault 2007, Maus 2008, 2010). To obtain an accurate model of internal magnetic field over some region, i.e., a regional model, it is desirable to combine information from ground (observatories, repeat stations, ground surveys), aeromagnetic, and satellite measurements. These complementary data sets (in spectral information content) are used to produce realistic regional or only lithospheric field models, with high spatial resolution (Haines 1985, De Santis et al. 1990, De Santis 1991; Thébault et al. 2006, Korte and Thébault 2007).

There were developed several mathematical approaches by now for modeling of the regional field. Brief review of the earlier methods, such as rectangular harmonic analysis or Taylor polynomial analysis, are given in Haines (1985) and references therein. Haines (1985) introduced spherical cap harmonic analysis (SCHA). In this method, the general solution in spherical coordinates of Laplace's equation over the spherical cap includes associated Legendre functions of integral order and non-integral degree. Further improvements and also some reasonable simplifications of SCHA are given by translated origin of spherical cap harmonic analysis (TOSCA, De Santis 1991), and adjusted spherical cap harmonic analysis (ASHA, De Santis 1992). Based on SCHA, the revised spherical cap harmonic analysis (R-SCHA) was introduced (Thébault et al. 2006), in order to combine ground, aeromagnetic, and satellite data. The main advantage of this method is improved altitude dependence, and it gives a full three-dimensional description of vector lithospheric field, from ground to satellite altitude (Thébault et al. 2006, Korte and Thébault 2007).

In this case study the three techniques were used for regional modeling: Taylor polynomial analysis (Meloni et al. 1994, Chiappini et al. 1999, Gu et al. 2006), Adjusted Spherical Harmonic Analysis (De Santis 1992, Chiappini et al. 1999, Kovács et al. 2011), and Spherical Harmonic Analysis. The first two methods were applied on ground data from Croatia and surrounding countries at 2009.5 epoch, while the third method refers to global enhanced magnetic model (EMM) data at 2009.5. This high-degree global model up to degree and order of 720 is derived from ground surveys, marine, aeromagnetic, and satellite data (Maus 2010). The core field is described by degrees of 1-15, and the static lithospheric field by degrees of 16-720. 
Relaying on definitions given in Thébault et al. (2010), the term crustal instead of lithospheric field will be used in this paper, since the satellite data are not used in the study. The crustal field will represent all the contributions from magnetized rocks that are below the Curie temperature, i.e., the Curie depth is a magnetic boundary, varying from place to place (Thébault et al. 2010). The aim of this work was to derive the most accurate regional model of geomagnetic components by using different techniques, and to estimate the crustal field at Croatian repeat stations. In Section 2 the descriptions of data and techniques are given, and in Section 3 the results with discussion are presented, which are followed with conclusions in the ending section.

\section{DATA SETS AND USED GEOMAGNETIC MODELS}

\subsection{Data}

For modelling the regional field over Croatia, the values of $X, Y$, and $Z$ geomagnetic components for the epoch 2009.5 were used: 10 Croatian repeat stations, 88 Croatian ground survey stations, 8 geomagnetic observatories, 44 repeat stations from surrounding countries, and 2 additional points. These 2 points were derived from EMM up to degree of 720, to fill the gap on the eastern part of the region of interest. Positions of all the sites are displayed in Fig. 1. The reference point, i.e., the pole of regional models (Sections 2.3 and 2.4) is denoted by a cross, and its coordinates are $\varphi_{0}=44.6^{\circ} \mathrm{N}$ and $\lambda_{0}=16.5^{\circ} \mathrm{E}$. Considering the points in Fig. $1 \mathrm{a}$, the maximal distance between the first neighbours is $143 \mathrm{~km}$, the minimal is $28 \mathrm{~km}$, and the average

(a)
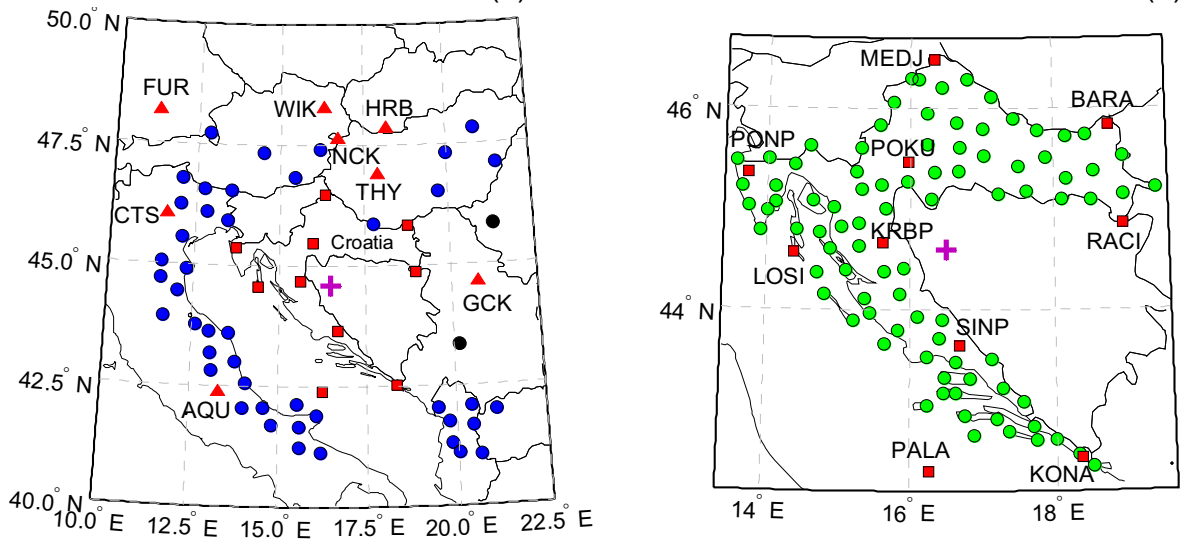

Fig. 1. The positions of sites used in this study: (a) Croatian repeat stations (squares), observatories (triangles), European repeat stations (blue circles), and two EMM points (black circles, see text); the pole position is denoted by a purple cross; and (b) Croatian repeat stations (squares) and Croatian ground survey stations (circles). 
is $(67 \pm 31) \mathrm{km}$. The same statistics for Croatian repeat stations is: $171 \mathrm{~km}$, $94 \mathrm{~km}$, and $(118 \pm 26) \mathrm{km}$, and for the Basic Geomagnetic Network of the Republic of Croatia shown in Fig. 1b: 65, 5, and (24 \pm 8$) \mathrm{km}$.

The data from European observatories (annual means) and repeat stations were attained at British Geological Survey web site (http://www. geomag.bgs.ac.uk). The used observatories are: L'Aquila (AQU), Castello Tesino (CTS), Fürstenfeldbruck (FUR), Grocka (GCK), Hurbanovo (HRB), Nagycenk (NCK), Tihany (THY), and Wien Kobenzl (WIK). The repeat stations are from: Albania, Austria, Germany, Hungary, Italy, and FYR of Macedonia.

The Croatian repeat station data for 2009.5 were reduced by AQU, GCK, and THY observatories, by the method described in Brkić et al. (2012). The ground surveys of 88 points were performed in 2008, 2009, and 2010 (Šugar et al. 2013). The station observations reduced primarily to 2008.5 and 2010.5 were corrected finally to 2009.5 epoch, by using the second-order Taylor polynomial (see Section 2.3) of the annual change of geomagnetic components (2008.5-2009.5 and 2009.5-2010.5, respectively), derived from Croatian repeat stations and surrounding observatory data from 2008.5, 2009.5, and 2010.5 (Vujić et al. 2013).

\subsection{Adjustment due to the long-term external variations' influence}

Since the long-term external variations and their induced effects do not average out entirely in observatory annual means, the same is valid for the repeat station and ground survey annual means (see Korte and Thébault (2007) and references therein). The latter two eventually suffer additionally from shortterm temporal variations influence, due to the non-perfect data reduction. However, it is possible to use an empirical method for estimating the influence of the long-term external variations, as proposed by Korte and Thébault (2007). The basic assumption is that the external influence is homogeneous over some region, and it can be estimated by using the records of several observatories inside the region of interest.

In this work the annual means from FUR, GCK, and THY for 2007.52010.5 epochs were used. The same procedure was used for all three observatories in establishing the long-term external influence, and their averaged values of adjustments for $X, Y$, and $Z$ were taken as corrections for all the stations in the study. First, the values predicted by EMM of degree up to 720 were subtracted from observatory annual means. Further, the constant offsets (present due to local magnetic effects) and the linear trends (due to EMM misfit) computed for the studied time period (2007.5-2010.5), were subtracted from the time-series of the elements' annual means. The remainder is the estimation of the long-term external influence at particular obser- 
vatory, while their means represent the estimation of the regional external influence. These adjustments, e.g., for 2009.5 epoch in $X, Y$, and $Z$ were 3.4, -1.1 , and $-2.5 \mathrm{nT}$, respectively. Finally, these corrections can be subtracted from sites' annual means, to obtain the final values as input for modeling.

\subsection{Taylor polynomial analysis (PA)}

The normal geomagnetic field is the mathematical estimation of a combination of the crustal and core field over a limited region. In order to describe the normal field over the investigated region, the Taylor polynomial over geographic latitude $\varphi$ and longitude $\lambda$ of the form (Meloni et al. 1994, Chiappini et al. 1999, Gu et al. 2006) was used:

$$
E(\varphi, \lambda)=\sum_{j=0}^{P} \sum_{i=0}^{P-j} a_{j i}\left(\varphi-\varphi_{0}\right)^{j}\left(\lambda-\lambda_{0}\right)^{i},
$$

where $E$ is a geomagnetic element, $P$ is the polynomial order $(P=2$, in our case), $a_{j i}$ are coefficients (their count is 18 ), $\varphi_{0}=44.6^{\circ}$ and $\lambda_{0}=16.5^{\circ}$. Such polynomial model does not satisfy Laplace's equation for the scalar potential of the total field, but can be useful to deduce anomalous regions over some area (Meloni et al. 1994, Gu et al. 2006). The coefficients in Eq. 1 were derived by linear regression of the element values given by that equation to measurements. Here the inversion problem was solved by using the singular value decomposition of the problem matrix (Press et al. 1992).

To avoid the influence of the data from highly anomalous areas to the normal field, weighted regression (WE-fit) has been applied (Meloni et al. 1994), in an iterative manner. The weights for each station point were initially set to 1 . After the first set of normal field coefficients has been computed, the standard uncertainty $\delta=\left[\Sigma_{i} \mathrm{rsd}_{i}^{2} /(N-6)\right]^{0.5}$ may be obtained, where $\operatorname{rsd}_{i}$ are the residuals, and $N$ is the number of points that participate in regression. In the second regression the weight is set to 0 for the points where the residual were more than $2 \delta$ and to 1 otherwise. The regression and the successive weighting are carried out iteratively until all of the rsd become lower than $2 \delta$. In this manner, some sites from the original data set have been rejected. This is the Chauvenet's criterion of rejection.

Assuming the absence of the vertical electrical currents in the atmosphere, i.e., the curl-free condition in vertical direction, Tsubokawa (1952) proposed the constrained polynomial analysis (CPA) of the components, in which coefficients $a_{01}, a_{02}, a_{11}$ for $X$ component, and $a_{10}, a_{20}, a_{11}$ for $Y$ component depend on each other. Thus the number of coefficients reduces to 12 (Chiappini et al. 1999). 


\subsection{Adjusted spherical harmonic analysis (ASHA)}

In the case of current-free circumstances, the magnetic field is curl-free and can be expressed as the negative gradient of a scalar potential function $V$. For global scale, the potential function can be approached in terms of spherical coordinates $(r, \Theta, \Lambda)$ by spherical harmonic analysis (SHA) of the measured magnetic elements. In order to achieve good spatial representation of the field in a regional scale, Haines (1985) introduced the technique of spherical cap harmonic analysis (SCHA), proper to fit the potential to a spherical cap of half angle $\theta_{0}$.

A mathematically more simple algorithm, for not too large caps, was implemented by De Santis (1992) by re-scaling the $\left[0, \theta_{0}\right]$ parameter space to the hemisphere $\left[0,90^{\circ}\right]$. This is the adjusted spherical harmonic analysis (ASHA), where the potential is expanded according to trigonometric and Legendre functions $P_{k}^{m}$ of integer order $m$ and degree $k$ as:

$$
V(r, \Theta, \Lambda)=a \sum_{k=0}^{K_{\max }}\left(\frac{a}{r}\right)^{m_{k}+1} \sum_{m=0}^{k} P_{k}^{m}(\cos \Theta)\left(g_{k}^{m} \cos m \Lambda+h_{k}^{m} \sin m \Lambda\right),
$$

where $a$ is the radius of the reference sphere, $K_{\max }$ is the truncation degree of analysis, $n_{k}$ is a real number given by $n_{k} \approx s(k+0.5)-0.5$, with $s=90^{\circ} / \theta_{0}$, $g_{k}^{m}$ and $h_{k}^{m}$ are Gauss coefficients.

Equation 2 is valid in hemispherical frame, and one has to perform the transformations of coordinates and geomagnetic elements $X, Y, Z$ from geographical to hemispherical frame, where:

$$
\vec{B}_{\text {hem }}=\left(X_{\text {hem }}, Y_{\text {hem }}, Z_{\text {hem }}\right)=-\nabla V \text {. }
$$

This can be done in the following steps (De Santis 1992): first there are transformations from geographical to geocentrical frame, then to spherical cap frame with half angle $\theta_{0}$, which are followed by transformations to hemispherical frame. After finding elements' values in hemispherical frame, one has to perform the inverse transformations to obtain the corresponding values in geographical frame, i.e., to compare it to the measurements.

The transformations of coordinates from spherical cap frame $\left(r^{\prime}, \Theta^{\prime}, \Lambda^{\prime}\right)$ to hemispherical frame $(r, \Theta, \Lambda)$ are given by (De Santis 1992, Chiappini et al. 1999):

$$
r=r^{\prime}, \Lambda=\Lambda^{\prime}, \Theta=s \Theta^{\prime},
$$

and of the geomagnetic elements are:

$$
X_{\text {hem }}=X_{\text {cap }} / s, \quad Y_{\text {hem }}=Y_{\text {cap }} \sin \Theta^{\prime} / \sin \Theta, \quad Z_{\text {hem }}=Z_{\text {cap }} .
$$


The coefficients in Eq. 2 are derived by linear regression of $X, Y$, and $Z$ values derived from that equation, to measurements. In this case, the inversion problem was also determined by using the singular value decomposition of the problem matrix. It is known that some regional modelling techniques are more efficient when dealing with a residual field, because the errors incurred in truncating the series like in Eq. 2 are less emphasized (Thébault et al. 2006). That was the reason why the input values were annual means for 2009.5 subtracted by EMM up to degree of 15 .

\section{RESULTS AND DISCUSSIONS}

\subsection{The geomagnetic models}

By examining the sites' distribution (Fig. 1), one can notice that the density of measurement sites inside Croatia is higher than outside of it (except the western part, i.e. Italian stations). This fact was taken into account when considering which data to pick out as an input for deriving the regional model. In this work we have used three data sets, i.e., the three regional models over Croatia were derived: (i) data set A, when model input was Croatian and European repeat stations', observatories', and two additional $\operatorname{EMM}(720)$ points' data (shown in Fig. 1a); (ii) data set B, when only Croatian data (98 sites from Fig. 1b) were used as an input; and (iii) data set C, when all data from Fig. 1 were used (152 sites, i.e., data set A + data set B). The relevant parameter of the particular model misfit was the root-meansquare (rms) of residuals (rsd), where rsd = input-model, calculated on 98 Croatian sites (Fig. 1b). For example, a modelling technique which gave the smallest rms of rsd for a given data set (A, B or C), was taken as a Model (i.e., Model A, Model B, and Model C).

For deriving Model A, 64 points were used as input into PA, WE-fit PA, $\mathrm{CPA}, \mathrm{ASHA}$, and also global $\operatorname{EMM}(n)$ values were calculated at those sites, where $n=15$ or $n=720$. The rms of residuals out of 98 Croatian sites are presented in Fig. 2a. The smallest rms of residuals in $X$ and $Y$ are for ASHA modelling, with $K_{\max }=11$ and $\theta_{0}=16-20^{\circ}$, while $\operatorname{EMM}(720)$ provides the best fit in $Z$ component. In a case of 10 Croatian repeat stations, the smallest rms of residuals are for ASHA, in all three components (shown in Fig. 2a). The corresponding residuals of PA in $X, Y$, and $Z$ are $68.7,41.5$, and $86.9 \mathrm{nT}$, and the same quantities for $\operatorname{EMM}(720)$ are 59.5, 31.0, and $88.6 \mathrm{nT}$, respectively.

In Fig. $2 b$ and c the same results are displayed for data set $B$ and data set $\mathrm{C}$, respectively. It is obvious that ASHA modelling with the given parameters is the most accurate, in all three components. The corresponding rms of residuals are highest in $X$ and lowest in $Z$ component, and they are quite lower for Model B. Finally, Fig. 2d displays vector rms (square root of the 

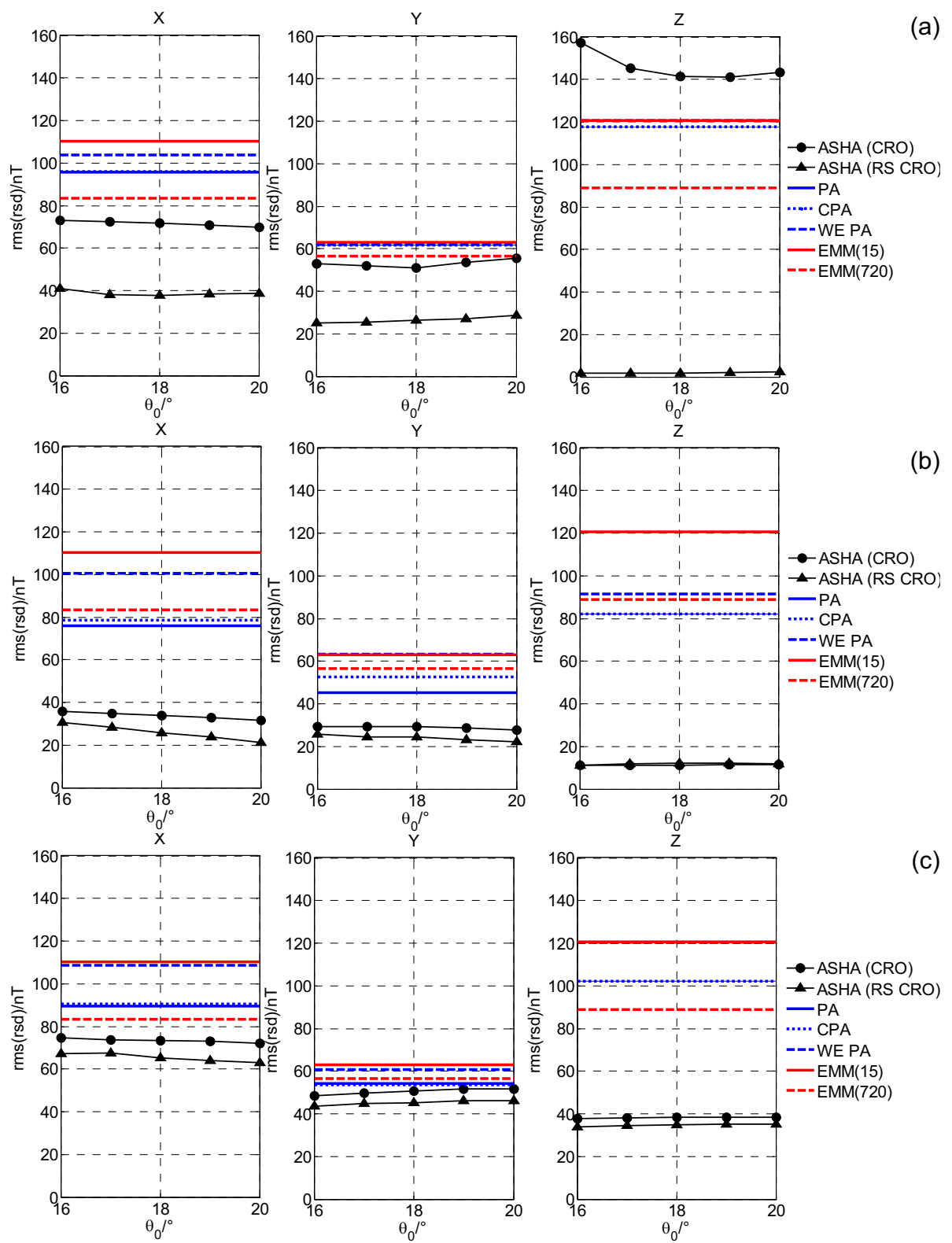

Fig. 2a-c. The root-mean-square (rms) of residuals of $X, Y$, and $Z$ components on 98 Croatian survey sites of three modelling techniques - Taylor polynomial (PA,WE PA, and CPA), EMM, and ASHA by using as an input: (a) the data set A, (b) the data set B, and (c) the data set C. "ASHA (RS CRO)" stands for ASHA rms of residuals calculated at 10 Croatian repeat stations, and "ASHA (CRO)" stands for ASHA rms of residuals calculated at 98 Croatian survey sites. 


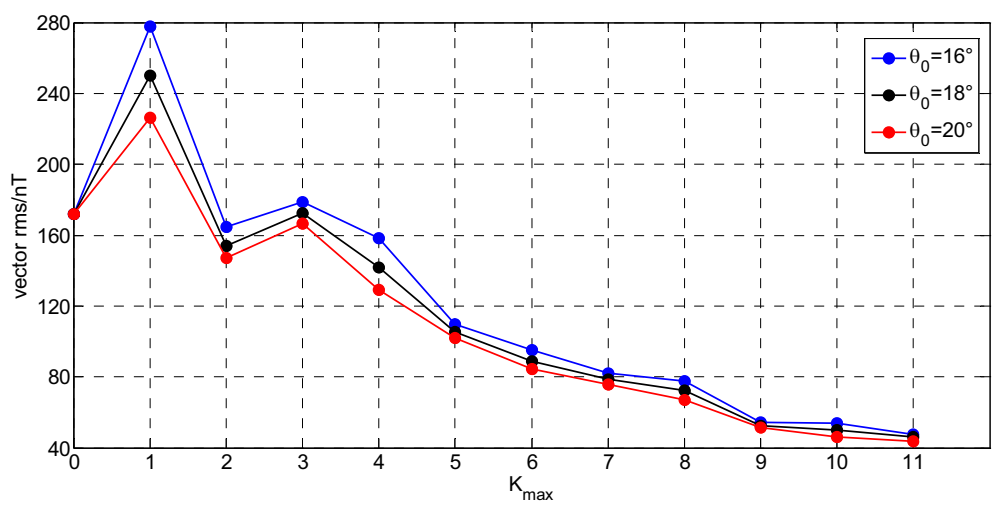

Fig. 2d. Vector rms (square root of the sum of squares of each single Cartesian component rms) when data set B was used as an input into ASHA modelling, as a function of $K_{\max }$. The parameter $\theta_{0}$ values are $16^{\circ}, 18^{\circ}$, and $20^{\circ}$.

sum of squares of each single component rms) of Model B, as a function of $K_{\max }=0-11$ with $\theta_{0}=16^{\circ}, 18^{\circ}$, and $20^{\circ}$ as parameter. One can notice an oscillatory behaviour of vector $\mathrm{rms}$ for $K_{\max }<3$, and a stabilization when $K_{\max }>8$.

By now one can conclude that Model B (by ASHA) is the most accurate over Croatian territory, but it was derived without data from the surrounding countries. Also, the rms of residuals in $X$ and $Y$ components are comparable between Model A and Model C, but in $Z$ component are significantly lower for Model C. Due to this, and a comparison between Model A and Model B results, it is obvious that it is necessary to have a denser network than ten Croatian repeat stations, and to carefully choose which surrounding sites could be included in modelling to obtain more accurate regional model over Croatia.

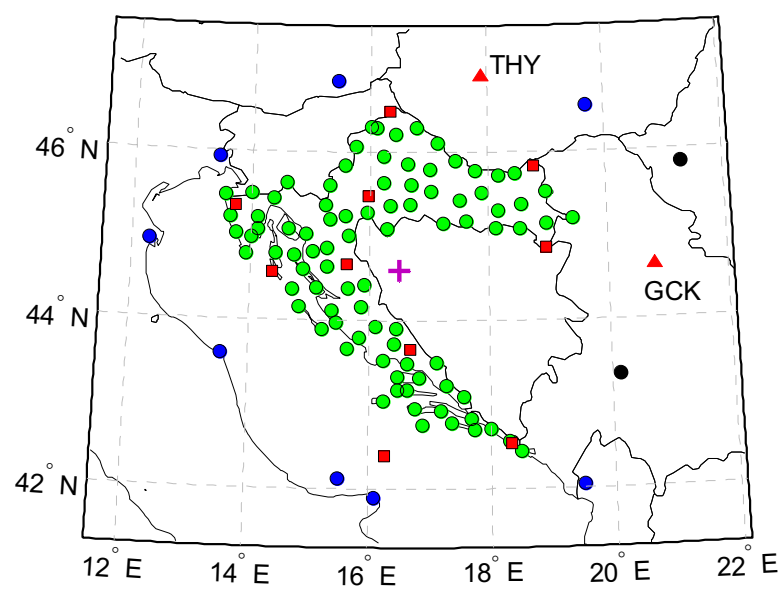

Fig. 3. The Croatian survey sites from Fig. 1, with the closest neighbours from the surrounding countries (data set C2). 
That was the reason why the additional two modifications of data set $\mathrm{C}$ have been done. The first one (data set C2) is the case when all 98 Croatian sites together with the twelve closest neighbours from the surrounding countries (Fig. 3) were used, and in the second one (data set C3) all Croatian stations together with the surrounding eight observatories were used. Those results are displayed in Table 1. The results for Model C2 are better than for Model C3 (it was stable up to $K_{\max }=10$ ) in rms sense, and they are comparable to Model B, either than Model A or C.

Table 1

The rms of residuals of $X, Y$, and $Z$, for data sets $\mathrm{C} 2$ and $\mathrm{C} 3$, by using the different modelling techniques and calculated on 98 Croatian sites (see text for details)

\begin{tabular}{|c|c|c|c|c|c|c|c|}
\hline \multicolumn{8}{|c|}{ rms of residuals $/ \mathrm{nT}$} \\
\hline Data set $\mathrm{C} 2$ & $X$ & $Y$ & $Z$ & Data set C3 & $X$ & $Y$ & $Z$ \\
\hline WE PA & 96.4 & 52.3 & 90.5 & WE PA & 92.5 & 52.7 & 96.3 \\
\hline PA & 80.4 & 49.8 & 87.6 & PA & 82.2 & 49.1 & 90.1 \\
\hline CPA & 81.9 & 51.4 & 87.6 & CPA & 84.0 & 49.3 & 90.1 \\
\hline $\mathrm{EMM}_{1}$ & 110.4 & 63.1 & 120.7 & $\mathrm{EMM}_{15}$ & 110.4 & 63.1 & 120.7 \\
\hline $\mathrm{EMM}_{720}$ & 83.5 & 56.5 & 88.8 & $\mathrm{EMM}_{720}$ & 83.5 & 56.5 & 88.8 \\
\hline $\begin{array}{c}\text { ASHA } \\
\left(K_{\max }=11, \theta_{0}=20^{\circ}\right)\end{array}$ & 33.0 & 32.9 & 20.1 & $\begin{array}{c}\text { ASHA } \\
\left(K_{\max }=10, \theta_{0}=20^{\circ}\right)\end{array}$ & 44.5 & 37.0 & 23.0 \\
\hline $\begin{array}{c}\text { ASHA } \\
\left(K_{\max }=11, \theta_{0}=16^{\circ}\right)\end{array}$ & 43.3 & 35.0 & 19.5 & $\begin{array}{c}\text { ASHA } \\
\left(K_{\max }=10, \theta_{0}=16^{\circ}\right)\end{array}$ & 50.3 & 37.8 & 21.8 \\
\hline
\end{tabular}

It is clear that the polynomial analysis has no way to compete with EMM and ASHA; the former kind of model has only 12-18 coefficients, while the latter kinds of models have from 144 (ASHA, $K_{\max }=11$ ) to 520560 of $\operatorname{EMM}(720)$ coefficients. In addition, $\operatorname{EMM}(720)$ is valid over all Earth's surface so to be compared with ASHA it should be normalized to the same area. The $16^{\circ}$ spherical cap surface is around 0.0194 of the entire Earth, so $\operatorname{EMM}(720)$ corresponds to 10083 coefficients over Croatia and surrounding area. This fact is reflected in the results of Table 1.

Finally, the Model B derived with ASHA $\left(K_{\max }=11, \theta_{0}=16^{\circ}\right)$ for crustal field, added to $\operatorname{EMM}(15)$ for core field, was taken as the regional model over Croatia for 2009.5 epoch. It corresponds approximately to a maximal spatial resolution of $300 \mathrm{~km}$. In Fig. 4 are the isolines of $X, Y$, and $Z$ components. Note that the distortion in the western part of Croatia in all three components is probably due to the Adriatic anomaly (Brkić et al. 2013), which 
was recognized by our regional model, at least in some of its long and intermediate wavelengths. Figure 5 shows the contours of Model B for crustal field, derived with ASHA $\left(K_{\max }=11, \theta_{0}=16^{\circ}\right)$, at the sea level for 2009.5 of $X, Y$, and $Z$ components, respectively.

(a)
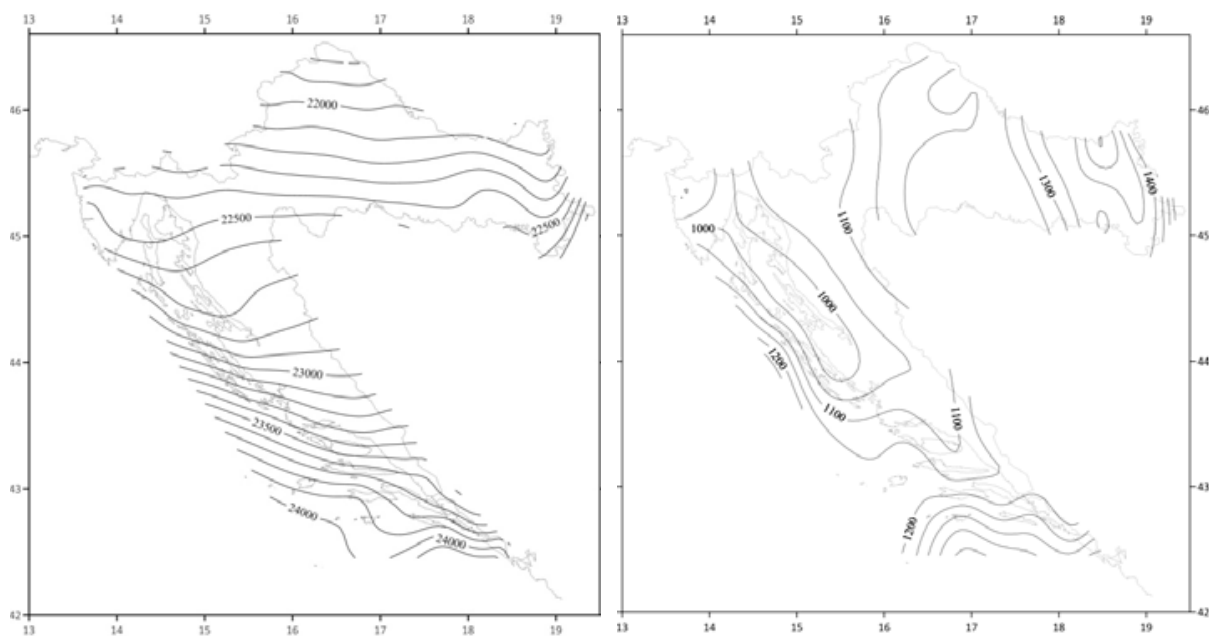

(c)

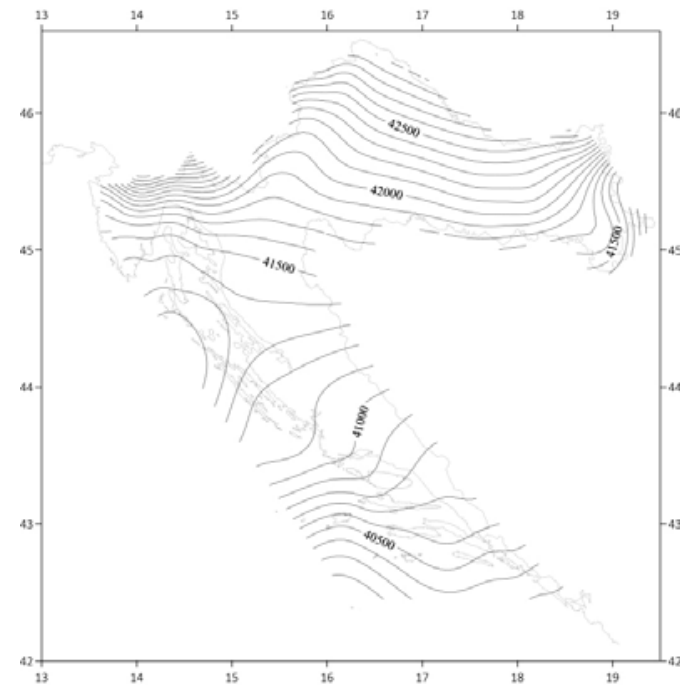

Fig. 4. The regional model of: (a) $X$ component, (b) $Y$ component, and (c) $Z$ component for 2009.5 at the sea level. The isolines are in $\mathrm{nT}$. 

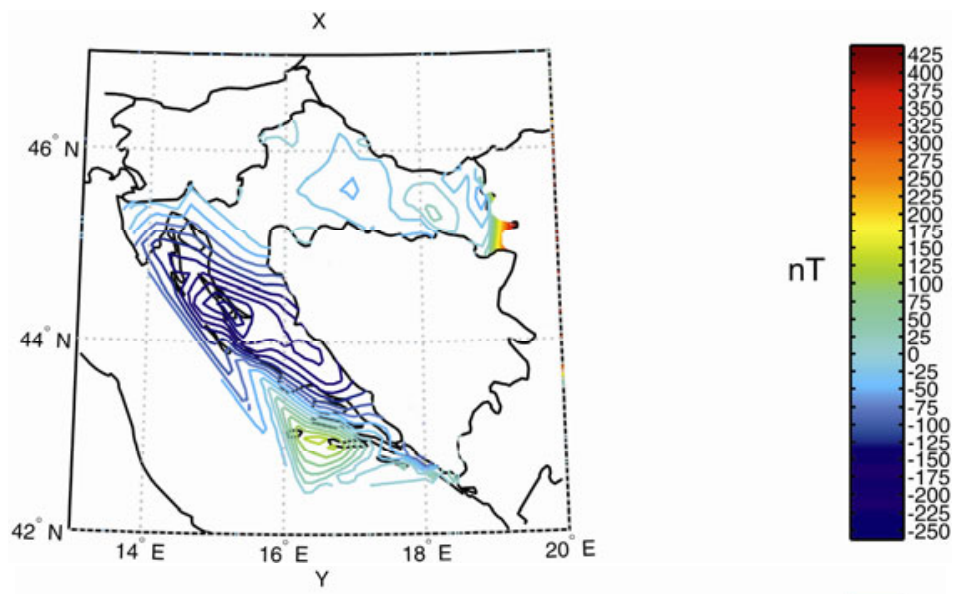

(a)
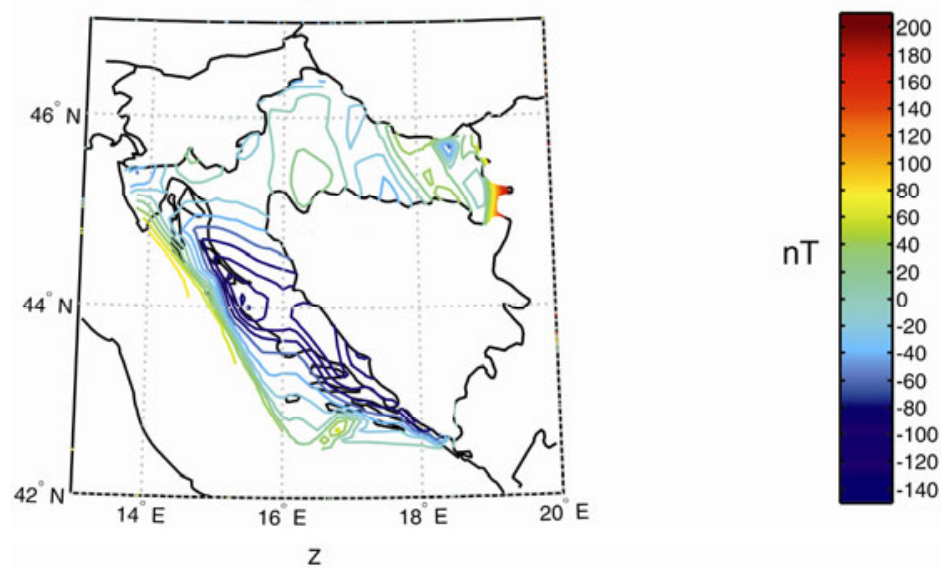

(b)
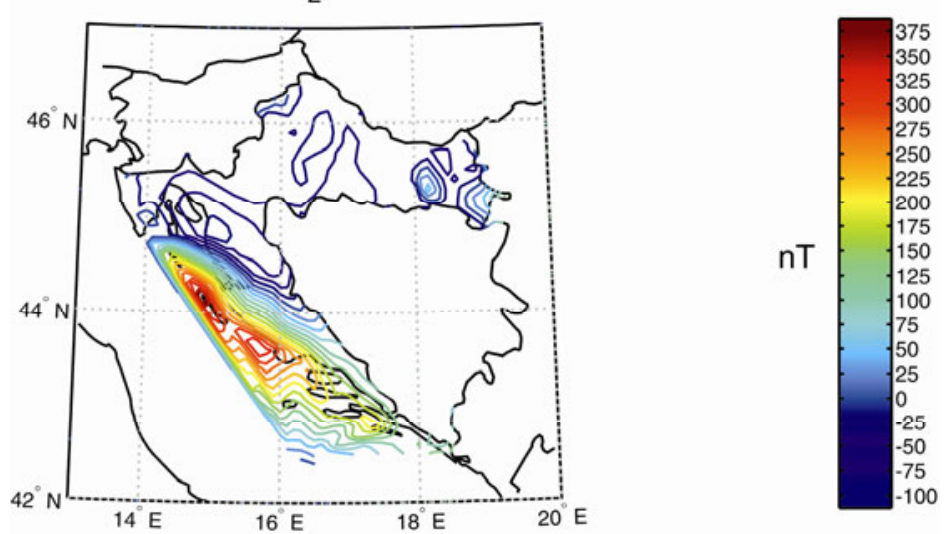

(c)

Fig. 5. The contours of: (a) $X$ component, (b) $Y$ component, and (c) $Z$ component of the Model B for crustal field, derived with ASHA $\left(K_{\max }=11, \theta_{0}=16^{\circ}\right)$, at the sea level for 2009.5. The isolines are in nT. 


\subsection{The crustal field at Croatian repeat stations}

For estimation of the crustal field at Croatian repeat stations from $X, Y$, and $Z$ values at epochs 2007.5-2010.5, the corresponding $\operatorname{EMM(15)~values,~i.e.,~}$ core field, were subtracted. The differences were the estimations of the crustal field, and the mean values over the given epochs were taken as a representative. This was done in two ways, with and without external field adjustment (Section 2.2). The results are presented in Fig. 6 (with external field adjustment) and Table 2. The higher crustal field values are at those stations that are on the Adriatic anomaly (LOSI and SINP). The root-mean-square of residuals of Model B (derived with ASHA) at Croatian repeat stations are 31.4, 25.3, and $10.8 \mathrm{nT}$ in $X, Y$, and $Z$, respectively, and the corresponding values derived by $\operatorname{EMM}(16-720)$, i.e., the crustal field estimations, are 59.5, 31.1 , and $88.3 \mathrm{nT}$, respectively.

The issue encountered was that the three stations do not have data for all of the four studied epochs. Stations LOSI and PALA were installed in 2008, but LOSI has to be removed due to contamination and destruction to the location in its vicinity in 2009. The similar was valid for station MEDJ in 2009, as reported in Brkić et al. (2012). Although the results were derived for all available epochs at LOSI ( 3 epochs) and MEDJ (4 epochs), there are high biases between the results for old and new locations, respectively. Therefore the results for these two stations are taken as means from 2009.5 and 2010.5 .

In Table 2 the standard deviations of the crustal field values are given, with and without external field correction for each epoch. One can notice that there is general decrease of standard deviations due to this correction, and the mean values (out of 10) of such decreases are $1.7 \mathrm{nT}$ in $X, 0.4 \mathrm{nT}$
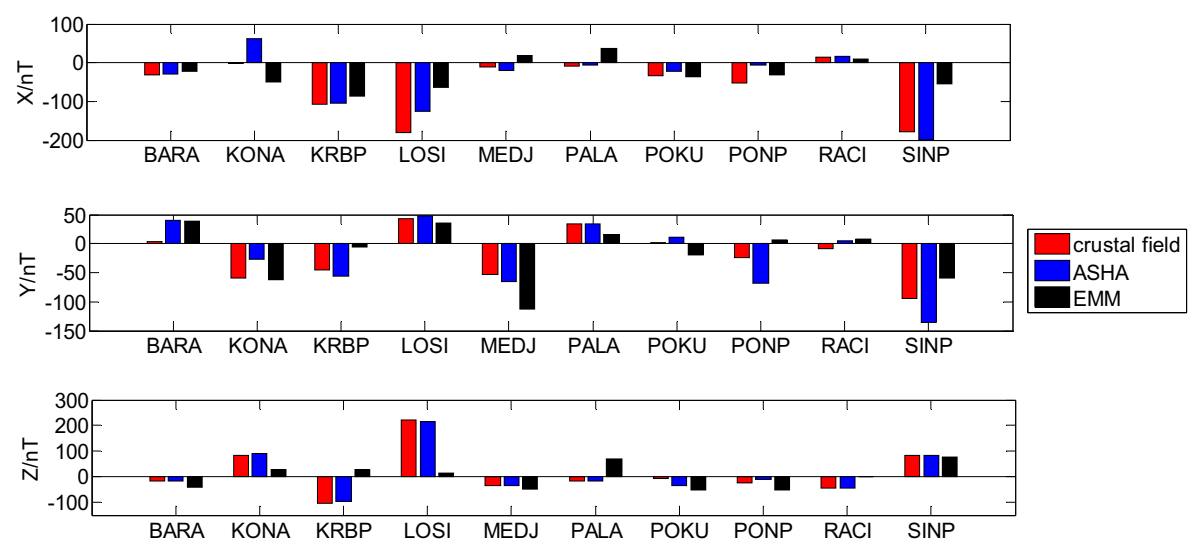

Fig. 6. The crustal field at Croatian repeat stations, in $X, Y$, and $Z$ components, together with ASHA (Model B, $K_{\max }=11, \theta_{0}=16^{\circ}$ ) and $\operatorname{EMM}(16-720)$ estimations. 
Table 2

The basic statistic of the crustal field estimations at Croatian repeat stations

\begin{tabular}{|c|c|c|c|c|c|c|c|}
\cline { 2 - 7 } \multicolumn{1}{c|}{} & $N_{\text {epoch }}$ & $\sigma_{X}$ & $\sigma_{Y}$ & $\sigma_{Z}$ & $\sigma_{X c}$ & $\sigma_{Y c}$ & $\sigma_{Z c}$ \\
\hline BARA & 4 & 3.0 & 2.4 & 2.5 & 1.6 & 2.3 & 2.3 \\
KONA & 4 & 3.8 & 2.8 & 2.2 & 1.9 & 2.3 & 1.1 \\
KRBP & 4 & 3.2 & 1.0 & 2.3 & 1.5 & 0.7 & 1.7 \\
LOSI & 2 & 3.3 & 1.4 & 1.7 & 0.7 & 0.1 & 1.1 \\
MEDJ & 2 & 2.6 & 2.0 & 1.5 & 1.4 & 0.7 & 1.3 \\
PALA & 3 & 3.6 & 0.7 & 3.5 & 2.5 & 1.1 & 2.3 \\
POKU & 4 & 2.8 & 2.1 & 1.4 & 0.8 & 1.4 & 1.7 \\
PONP & 4 & 3.9 & 2.7 & 3.0 & 2.4 & 2.3 & 2.4 \\
RACI & 4 & 2.8 & 2.4 & 1.5 & 1.3 & 2.6 & 1.1 \\
SINP & 4 & 2.9 & 2.5 & 2.6 & 0.7 & 2.7 & 2.4 \\
mean & - & 3.2 & 2.0 & 2.2 & 1.5 & 1.6 & 1.7 \\
\hline
\end{tabular}

Explanations: $N_{\text {epoch }}$ is a number of epochs used in the crustal field estimation, $\sigma$ and $\sigma_{c}$ are the standard deviations of the crustal field values (in nT), without and with external field correction, respectively.

in $Y$, and $0.5 \mathrm{nT}$ in $Z$. The same decreases (out of 3) for observatories FUR, GCK, and THY are $1.6 \mathrm{nT}$ in $X, 0.3 \mathrm{nT}$ in $Y$, and $0.6 \mathrm{nT}$ in $Z$. The crustal field at observatory FUR was estimated to be: $-15.0 \pm 1.2 \mathrm{nT}$ in $X$, $-5.7 \pm 0.5 \mathrm{nT}$ in $Y$, and $15.6 \pm 1.9 \mathrm{nT}$ in $Z$, which is in good agreement with the values given in Korte and Thébault (2007).

\section{CONCLUSIONS}

The Croatian repeat stations' and ground survey data of $X, Y$, and $Z$ components reduced at 2009.5 epoch, together with surrounding observatories' and repeat stations' data at the same epoch, were used to obtain the most accurate geomagnetic model over Croatia. Generally the input data into regional modelling can suffer from several errors: measurement errors, reduction errors (both due to the short-term and long-term external field variations), and a core field model misfit. In this study different models were derived, depending on input data, and the different techniques were used for modelling: Taylor polynomial analysis, spherical harmonic analysis, and adjusted spherical harmonic analysis.

The latter was used by changing its two parameters (truncation degree and spherical cap half-angle), in order to obtain the minimal and stable rootmean-square values of model residuals. It was obtained that the most accurate model was for $K_{\max }=11, \theta_{0}=16-20^{\circ}$, with the rms of residuals at Croatian sites less than $36 \mathrm{nT}$ in $X, 30 \mathrm{nT}$ in $Y$, and $12 \mathrm{nT}$ in $Z$, and when only 
Croatian Basic Geomagnetic Network (98) data were used as modeling input. Actually this model is the regional crustal field model, and to obtain a model of internal field one has to add the values from a core field model. Finally, the isolines of geomagnetic components at the sea level were produced.

By using the repeat station data in the interval 2007.5-2010.5, and a core field model EMM, the crustal field at Croatian stations was estimated. The higher values were found for the sites which lie on the regional Adriatic anomaly, and this anomaly was clearly recognized by the regional model. It was also obtained that the empirical corrections to the geomagnetic components' annual means, due to the long-term external field variations, generally resulted in the decrease of the standard deviations of crustal field values, obtained for different epoch.

Acknowledgments. The authors acknowledge the Institute for Research and Development of Defence Systems of Ministry of Defence, the State Geodetic Administration, and the Ministry of Science, Education and Sports of the Republic of Croatia. The authors are also grateful to the Institutions which provide the geomagnetic observatories' and repeat stations' data to the British Geological Survey web site. The authors are very thankful to Prof. A. De Santis for his constructive comments that improved the manuscript.

\section{References}

Brkić, M., D. Šugar, M. Pavasović, E. Vujić, and E. Jungwirth (2012), Croatian geomagnetic field maps for 2008.5 epoch, Ann. Geophys. 55, 6, 1061-1069, DOI: $10.4401 /$ ag-5395.

Brkić, M., M. Varga, and V. Poslončec-Petrić (2013), Geomagnetic field maps for the 2009.5 epoch. In: M. Brkić (ed.), Basic Geomagnetic Network of the Republic of Croatia 2004-2012, with Geomagnetic Field Maps at 2009.5 Epoch, Croatian State Geodetic Administration, Zagreb, 33-38 (in Croatian).

Chiappini, M., A. De Santis, G. Dominici, and J.M. Torta (1999), A normal reference field for the Ionian sea area, Phys. Chem. Earth A 24, 5, 433-438, DOI: 10.1016/S1464-1895(99)00071-X.

De Santis, A. (1991), Translated origin spherical cap harmonic analysis, Geophys. $J$. Int. 106, 1, 253-263, DOI: 10.1111/j.1365-246X.1991.tb04615.x. 
De Santis, A. (1992), Conventional spherical harmonic analysis for regional modelling of the geomagnetic field, Geophys. Res. Lett. 19, 10, 1065-1067, DOI: $10.1029 / 92$ GL01068.

De Santis, A., O. Battelli, and D.J. Kerridge (1990), Spherical cap harmonic analysis applied to regional field modelling for Italy, J. Geomag. Geoelectr. 42, 9, 1019-1036, DOI: 10.5636/jgg.42.1019.

Gu, Z., Z. Zhan, J. Gao, W. Han, Z. An, T. Yao, and B. Chen (2006), Geomagnetic survey and geomagnetic model research in China, Earth Planets Space 58, 6, 741-750, DOI: 10.1186/BF03351977.

Haines, G.V. (1985), Spherical cap harmonic analysis, J. Geophys. Res. 90, B3, 2583-2591, DOI: 10.1029/JB090iB03p02583.

Korte, M., and E. Thébault (2007), Geomagnetic repeat station crustal biases and vectorial anomaly maps for Germany, Geophys. J. Int. 170, 1, 81-92, DOI: 10.1111/j.1365-246X.2007.03387.x.

Kovács, P., E. Vujić, A. Csontos, M. Brkić, B. Heilig, and A. Koppán (2011), Regional magnetic field model for Croatia and Hungary. In: Abstr. 6th Congr. Balkan Geophysical Society "Magnetics and EM", 3-6 October 2011, Budapest, Hungary.

Maus, S. (2008), The geomagnetic power spectrum, Geophys. J. Int. 174, 1, $135-$ 142, DOI: 10.1111/j.1365-246X.2008.03820.x.

Maus, S. (2010), An ellipsoidal harmonic representation of Earth's lithospheric magnetic field to degree and order 720, Geochem. Geophys. Geosyst. 11, 6, Q06015, DOI: 10.1029/2010GC003026.

Meloni, A., O. Battelli, A. De Santis, and G. Dominici (1994), The 1990.0 magnetic repeat station survey and normal reference fields for Italy, Ann. Geophys. 37, 5, 949-967.

Press, W.H., S.A. Teukolsky, W.T. Vetterling, and B.P. Flannery (1992), Numerical Recipes in Fortran 77: The Art of Scientific Computing, Cambridge University Press, New York.

Šugar, D., M. Rezo, M. Pavasović, M. Šljivarić, and O. Bjelotomić (2013), Basic Geomagnetic Network of the Republic of Croatia and geomagnetic surveys in 2004-2012. In: M. Brkić (ed.), Basic Geomagnetic Network of the Republic of Croatia 2004-2012, with Geomagnetic Field Maps at 2009.5 Epoch, Croatian State Geodetic Administration, Zagreb, 10-20 (in Croatian).

Thébault, E., M. Mandea, and J.J. Schott (2006), Modeling the lithospheric magnetic field over France by means of revised spherical cap harmonic analysis (R-SCHA), J. Geophys. Res. 111, B5, 949-967, DOI: 10.1029/ 2005JB004110.

Thébault, E., M. Purucker, K.A. Whaler, B. Langlais, and T.J. Sabaka (2010), The Magnetic field of the Earth's lithosphere, Space Sci. Rev. 155, 1-4, 95-127, DOI: $10.1007 / \mathrm{s} 11214-010-9667-6$. 
Tsubokawa, I. (1952), Reduction of the results obtained by the magnetic survey of Japan (1948-51) to the epoch 1950.0 and reduction of the empirical formulae expressing the magnetic elements, Bull. Geogr. Surv. Inst. 3, 1-29.

Vujić, E., M. Brkić, and O. Bjelotomić (2013), Reduction methods, normal reference field and annual changes in 2004-2012. In: M. Brkić (ed.), Basic Geomagnetic Network of the Republic of Croatia 2004-2012, with Geomagnetic Field Maps at 2009.5 Epoch, Croatian State Geodetic Administration, Zagreb, 21-32 (in Croatian).

Received 28 March 2014

Received in revised form 15 September 2014 Accepted 5 January 2015 